\title{
Twofold Personalized Feedback Mechanism for Social Network Consensus by Uninorm Interval Trust Propagation
}

\author{
Jian Wu, Senior Member, IEEE, Sha Wang Francisco Chiclana, and Enrique Herrera-Viedma. \\ Fellow, IEEE
}

\begin{abstract}
A twofold personalized feedback mechanism is established for consensus reaching in social network group decisionmaking (SN-GDM). It consists of two stages: (1) generating the trusted recommendation advice for individuals; and (2) producing personalized adoption coefficient for reducing unnecessary adjustment costs. This is achieved by means of a uninorm interval-valued trust propagation operator to obtain indirect trust. The trust relationship is used to generate personalized recommendation advice based on the principle of 'a recommendation being more acceptable the higher the level of trust it derives from'. An optimization model is built to minimise the total adjustment cost of reaching consensus by determining personalized feedback adoption coefficient based on individuals' consensus levels. Consequently, the proposed twofold personalized feedback mechanism achieves a balance between group consensus and individual personality. An example to demonstrate how the proposed twofold personalized feedback mechanism works is included, which is also used to show its rationality by comparison with the traditional feedback mechanism in GDM.
\end{abstract}

Index Terms-Group decision making; Consensus; Social network; Uninorm interval trust propagation; Personalized feedback; Minimum cost.

\section{INTRODUCTION}

$\mathbf{G}$ ROUP decision making (GDM) involves a group of experts with preferences on multiple alternatives under a series of criteria, which are aggregated into a collective preference from which a group solution is derived [1]-[5]. Experts from multiple organizations usually have different backgrounds and knowledge, which may lead to different views on decisionmaking issues, and even non-cooperative behaviors due to their different attitudes and interests [6]; thus, GDM usually deals with inconsistent opinions hindering the way to arrive at a collective solution [7]-[9]. Consequently, how to reach consensus is a challenge that requires tackling theoretically in any GDM problem resolution [10]-[14]. A basic framework

Jian Wu and Sha Wang are with the School of Economics and Management, Shanghai Maritime University, Shanghai 201306, China (jyajian@163.com; wangsha_5323@126.com).

Francisco Chiclana is with the Institute of Artificial Intelligence (IAI), Faculty of Computing, Engineering and Media, De Montfort University, Leicester, UK and with the Andalusian Research Institute on Data Science and Computational Intelligence (DaSCI), University of Granada, Spain (chiclana@dmu.ac.uk).

Enrique Herrera-Viedma is the Andalusian Research Institute on Data Science and Computational Intelligence (DaSCI), University of Granada, Spain and with the School of Business Administration, Southwestern University of Finance and Economics, Chengdu 610074, China(viedma@decsai.ugr.es). of group consensus process involving a feedback mechanism, which generates advice on how experts in the group should modify their preferences to arrive at the threshold value of group consensus, has been proposed and applied in [15][20]. Also, a comprehensive work of feedback mechanism paradigms is investigated in [21]. Hence, the feedback mechanism plays a key role in group consensus process because its modelling rationality will affect the inconsistent decision maker acceptance or not of the generated advice.

A classic feedback mechanism in GDM derives feedback advices using a feedback coefficient that is fixed beforehand by subjective preference [16], [17] and that is common to all inconsistent experts. This could be seen as a reaching consensus process that enforces inconsistent experts to adopt feedback advices with excessive adjustment costs. The excessive adjustment cost was address by Wu et al. [22] with a 'nonpersonalized' feedback mechanism with minimum adjustment cost. In general, these types of traditional feedback mechanism are helpful to reach consensus. However, they still suffer from the drawback of generating feedback advices using experts' preferences only [11] and ignoring experts' trust relationships [23]-[25]. Consequently, inconsistent experts are not able to obtain personalized advices in the traditional feedback mechanisms and they are forced to homogeneously arrive to a 'group center' in order to reach group consensus. In fact, individuals have different trusting relationships with the opinions of other members of the group. Thus, it is necessary to develop a personalized feedback mechanism by personal trusted opinion and study is effective in reducing adjustment cost for consensus.

People usually rely on the advice/recommendations from their friends and acquaintances, nowadays via social network platforms, for changing their opinions [26]-[29]. From this perspective, an inconsistent expert may tend to be closer to the 'individual center' derived from his/her own social network than to the aforementioned 'group center'. Considering that trust relationship plays a very important role in social networks[30], and is regarded as a useful resource for generating advice in feedback mechanism [31]-[34]. Therefore, the first objective of this article is to develop a twofold personalized feedback mechanism for social network consensus, which (1) uses trust relationship to generate the recommendation advice for individuals and (2) determines feedback coefficients by minimizing the adjustment cost for reaching consensus. Consequently, an inconsistent expert can know which recom- 
mendation advice he/she trusts according to their personality and how to implement them to satisfy the group consensus aim.

In social network, every individual may not have a direct trust relationship with other individual, i.e. the network may be incomplete. Trust propagation operators have been developed to make a complete trust relationship network if necessary [35], [36]. Victor et al. [32] proposed t-norms and t-conorms operators to propagate trust and distrust, respectively. $\mathrm{Wu}$ et al. [15] investigated the use of uninorm operators to propagate trust and distrust information simultaneously. Anyway, the value of trust and distrust is to be precisely expressed with numbers, which means that these approaches are not suitable to deal with trust/distrust uncertain manifestations [37]-[40]. This is addressed here with the investigation of the uninorm interval-valued trust propagation operator. Especially,the opinions of experts are expressed as interval values, which will be consistent with trust propagation information.

The rest of the article is set out as follows: Section II introduces the concept of interval-valued trust function, and their operator is investigated in Section III. Section IV proposes the twofold personalized feedback mechanism. An example is illustrated in Section V. Section VI is devoted to a comparative analysis between the traditional feedback mechanisms and proposed mechanism, while conclusions are drawn in Section VII.

\section{PRELIMINARIES}

In this section, some basic concepts associated to social network analysis (SNA) and the uninorm propagation operator are introduced.

\section{A. Interval-valued trust function}

An interval-valued trust function (IVTF) is represented as [17]:

$$
\operatorname{IVTF} \Omega=\{\tilde{r}=(\tilde{t}, \tilde{d}) \mid \tilde{t}, \tilde{d} \in \mathcal{C}([0,1])\}
$$

Where $\tilde{r}$ is a tuple, the first component $\tilde{t}$ is a trust degree and the second component $\tilde{d}$ is a distrust degree. $\mathcal{C}([0,1])$ is the set of closed subintervals of set $[0,1]$ :

$$
\mathcal{C}([0,1])=\left\{\left[x^{-}, x^{+}\right] \mid 0 \leq x^{-} \leq x^{+} \leq 1\right\} .
$$

The interval analysis as described in [41] will be used herein.

\section{B. Uninorm trust propagation operator}

A uninorm operator $U$ [42] is a mapping $U:[0,1]^{2} \longrightarrow$ $[0,1]$ having the following properties:

1) Commutativity: $U(x, y)=U(y, x)$

2) Monotonicity: $U\left(x_{1}, y\right) \leq U\left(x_{2}, y\right)$ if $x_{1} \leq x_{2}$.

3) Associativity: $U(x, U(y, z))=U(U(x, y), z)$

4) Identity element: $\exists e \in[0,1]: \forall x \in[0,1], U(x, e)=x$

Symmetric aggregative operators are uninorm operators with a representation based on a single variable function. In particular, the below cross ratio uniorm (identity element $e=0.5$ ) was used in [43] for a transitivity based estimation of unknown preference values in incomplete preference relations:

$$
U(x, y)=\left\{\begin{array}{l}
0, \quad(x, y) \in\{(0,1),(1,0)\} \\
\frac{x y}{x y+(1-x)(1-y)}, \text { otherwise. }
\end{array}\right.
$$

This idea was taking forward in [15] to propagate trust/distrust with uninorms in incomplete social networks:

$$
P_{U}\left(\zeta_{1}, \zeta_{2}\right)=\left\{\begin{array}{l}
(0,1), i f \zeta_{1} \vee \zeta_{2}=(0,1) \\
\zeta_{i}, i f \zeta_{j}=(1,0) ; i \neq j ; i, j \in\{1,2\} \\
\left(U\left(t_{1}, t_{2}\right), U\left(t_{1}, d_{2}\right)\right), \text { otherwise }
\end{array}\right.
$$

being $\zeta_{1}=\left(t_{1}, d_{1}\right), \zeta_{2}=\left(t_{2}, d_{2}\right)$ trust functions with crisp numerical trust/distrust information.

\section{UNINORM INTERVAL-VALUED TRUST PROPAGATION}

Uninorm operator is expanded to interval-valued inputs in this section for trust propagation. In particular the cross-ratio uniforms is fully developed.

\section{A. Interval-valued Uninorm Operator}

Let $\tilde{x}=\left[x^{-}, x^{+}\right], \tilde{y}=\left[y^{-}, y^{+}\right] \in \mathcal{C}([0,1])$ be two interval numbers. Given a uninorm operator $U:[0,1]^{2} \longrightarrow[0,1]$, the following operator $\tilde{U}: \mathcal{C}([0,1]) \times \mathcal{C}([0,1]) \rightarrow \mathcal{C}([0,1])$ can be defined:

$$
\tilde{U}(\tilde{x}, \tilde{y})=\left[U\left(x^{-}, y^{-}\right), U\left(x^{+}, y^{+}\right)\right]
$$

a) Operator $\tilde{U}$ is well defined: Uninorm operator $U$ is monotonic and therefore it is

$U\left(x^{-}, y^{-}\right) \leq U\left(x^{+}, y^{+}\right) \Longrightarrow\left[U\left(x^{-}, y^{-}\right), U\left(x^{+}, y^{+}\right)\right] \in \mathcal{C}([0,1])$.

b) Operator $\tilde{U}$ is commutative.: Uninorm operator $U$ is commutative and therefore it is

$$
\begin{aligned}
\tilde{U}(\tilde{x}, \tilde{y}) & =\left[U\left(x^{-}, y^{-}\right), U\left(x^{+}, y^{+}\right)\right] \\
& =\left[U\left(y^{-}, x^{-}\right), U\left(y^{+}, x^{+}\right)\right] \\
& =\tilde{U}(\tilde{y}, \tilde{x}) .
\end{aligned}
$$

c) Operator $\tilde{U}$ is associative: Uninorm operator $U$ is associative and therefore it is

$$
\begin{aligned}
\tilde{U}(\tilde{U}(\tilde{x}, \tilde{y}), \tilde{z}) & =\tilde{U}\left(\left[U\left(x^{-}, y^{-}\right), U\left(x^{+}, y^{+}\right)\right],\left[z^{-}, z^{+}\right]\right) \\
& =\left[U\left(U\left(x^{-}, y^{-}\right), z^{-}\right), U\left(U\left(x^{+}, y^{+}\right), z^{+}\right)\right] \\
& =\left[U\left(x^{-}, U\left(y^{-}, z^{-}\right)\right), U\left(x^{+}, U\left(y^{+}, z^{+}\right)\right)\right] \\
& =\tilde{U}\left(\left[x^{-}, x^{+}\right],\left[U\left(y^{-}, z^{-}\right), U\left(y^{+}, z^{+}\right)\right]\right) \\
& =\tilde{U}(\tilde{x}, \tilde{U}(\tilde{y}, \tilde{z}))
\end{aligned}
$$

d) Operator $\tilde{U}$ identity element: Let $e \in[0,1]$ be the identity element of the uninorm operator $U$, and define $\tilde{e}=$ $[e, e]$. Then we have the following:

$$
\tilde{U}(\tilde{x}, \tilde{e})=\left[U\left(x^{-}, e\right), U\left(x^{+}, e\right)\right]=\left[x^{-}, x^{+}\right]=\tilde{x}
$$

Thus, operator $\tilde{U}$ has $\tilde{e}$ as its identity element.

e) Operator $\tilde{U}$ monotonicity: To study operator $\tilde{U}$ monotonicity on $\mathcal{C}([0,1])$, we need to define an order relation on $\mathcal{C}([0,1])$. The ordering of real numbers in which monotonicity of uninorm operator is defined does not exist in the set of interval numbers. Many ordering relations have been proposed 
to order interval numbers [44], and therefore the monotonicity property of the operator $\tilde{U}$ will depend on the ordering of interval numbers used. An ordering relation of interval numbers $\preceq$ that verifies the property $\tilde{x} \preceq \tilde{y} \Rightarrow \tilde{U}(\tilde{x}, \tilde{z}) \preceq$ $\tilde{U}(\tilde{y}, \tilde{z}) \forall z \in \mathcal{C}([0,1])$ will make $\tilde{U}$ to be monotonic (wrt $\preceq)$, and therefore the interval number set in the unit interval (with ordering $\preceq$ ) is uniformly operated.Such ordering will be referred to as a U-monotonocity ordering and be denoted by $\preceq_{U}$. In particular, the ordering

$$
\tilde{x} \preceq_{U} \tilde{y} \Leftrightarrow x^{-} \leq y^{-} \wedge x^{+} \leq y^{+}
$$

is a U-ordering. Indeed,

$$
\begin{aligned}
& x^{-} \leq y^{-} \wedge x^{+} \leq y^{+} \Longrightarrow U\left(x^{-}, z^{-}\right) \leq U\left(y^{-}, z^{-}\right) \wedge \\
& U\left(x^{+}, z^{+}\right) \leq U\left(y^{+}, z^{+}\right) \forall \tilde{z}=\left[z^{-}, z^{+}\right] \in \mathcal{C}([0,1])
\end{aligned}
$$

We have proved in property a) that operator $\tilde{U}$ is well defined, and so it is

$$
\left[U\left(x^{-}, z^{-}\right), U\left(x^{+}, z^{+}\right)\right] \prec_{U}\left[U\left(y^{-}, z^{-}\right), U\left(y^{+}, z^{+}\right)\right],
$$

which implies that

$$
\tilde{U}(\tilde{x}, \tilde{z}) \prec \tilde{U}(\tilde{y}, \tilde{z}) \forall z \in \mathcal{C}([0,1]) .
$$

\section{B. Cross ratio uninorm interval trust propagation operator}

This subsection develops the cross ratio interval-valued trust propagation operator. Given a uninorm operator $U$, the interval-valued uninorm operator $\tilde{U}$ is constructed and therefore interval valued trust/distrust can be propagated by extending expression (3), as follows:

$$
\operatorname{IVP}_{\tilde{U}}\left(\tilde{\zeta}_{1}, \tilde{\zeta}_{2}\right)=\left\{\begin{array}{l}
([0,0],[1,1]), \tilde{\zeta}_{1} \vee \tilde{\zeta}_{2}=([0,0],[1,1]) \\
\tilde{\zeta}_{i}, \text { if } \tilde{\zeta}_{j}=([1,1],[0,0]) ; i \neq j ; i, j \in\{1,2\} \\
\left(\tilde{U}\left(\tilde{t}_{1}, \tilde{t}_{2}\right), \tilde{U}\left(\tilde{t}_{1}, \tilde{d}_{2}\right)\right), \text { otherwise }
\end{array}\right.
$$

The cross ratio uninorm interval trust propagation operator is well defined.We only need to verify that the third case output in expression (5)is an IVTF. Given two IVTFs $\tilde{\zeta}_{1}=\left(\tilde{t}_{1}, \tilde{d}_{1}\right)=$ $\left(\left[t_{1}^{-}, t_{1}^{+}\right],\left[d_{1}^{-}, d_{1}^{+}\right]\right), \tilde{\zeta}_{2}=\left(\tilde{t}_{2}, \tilde{d}_{2}\right)=\left(\left[t_{2}^{-}, t_{2}^{+}\right],\left[d_{2}^{-}, d_{2}^{+}\right]\right)$, because operator $\tilde{U}$ is well defined, it is

$$
\tilde{U}\left(\tilde{t}_{1}, \tilde{t}_{2}\right), \tilde{U}\left(\tilde{t}_{1}, \tilde{d}_{2}\right) \in \mathcal{C}([0,1]) \Longrightarrow \operatorname{IVP}_{\tilde{U}}\left(\tilde{\zeta}_{1}, \tilde{\zeta}_{2}\right) \in \Omega .
$$

In the following we prove the associativity property of $\operatorname{IVP}_{\tilde{U}}$, which is subsequently used to derive its expression when we have a social network trust/distrust path of length $n(\geq 3)$.

Proposition 1 (Associativity). Given IVTFs $\tilde{\zeta}_{i}=\left(\tilde{t}_{i}, \tilde{d}_{i}\right)(i=$ $1,2,3)$, it is:

$$
I V P_{\tilde{U}}\left(I V P_{\tilde{U}}\left(\tilde{\zeta}_{1}, \tilde{\zeta}_{2}\right), \tilde{\zeta}_{3}\right)=I V P_{\tilde{U}}\left(\tilde{\zeta}_{1}, I V P_{\tilde{U}}\left(\tilde{\zeta}_{2}, \tilde{\zeta}_{3}\right)\right) .
$$

Proof. 1) One of the IVTF is $([1,1],[0,0])$. Assume that $\tilde{\zeta}_{1}=([1,1],[0,0])$. On the one hand, we have

$$
\operatorname{IVP}_{\tilde{U}}\left(\tilde{\zeta}_{1}, \tilde{\zeta}_{2}\right)=\tilde{\zeta}_{2}
$$

and therefore it is

$$
\operatorname{IVP}_{\tilde{U}}\left(\operatorname{IVP}_{\tilde{U}}\left(\tilde{\zeta}_{1}, \tilde{\zeta}_{2}\right), \tilde{\zeta}_{3}\right)=\operatorname{IVP}_{\tilde{U}}\left(\tilde{\zeta}_{2}, \tilde{\zeta}_{3}\right) .
$$

On the other hand, it is:

$$
\operatorname{IVP}_{\tilde{U}}\left(\tilde{\zeta}_{1}, \operatorname{IVP}_{\tilde{U}}\left(\tilde{\zeta}_{2}, \tilde{\zeta}_{3}\right)\right)=\operatorname{IVP}_{\tilde{U}}\left(\tilde{\zeta}_{2}, \tilde{\zeta}_{3}\right)
$$

The proofs when $\tilde{\zeta}_{2}$ or $\tilde{\zeta}_{3}$ are $([1,1],[0,0])$ are the same. 2) One of the IVTF is $([0,0],[1,1])$. Assume that $\tilde{\zeta}_{1}=$ $([0,0],[1,1])$. On the one hand, we have

$$
\operatorname{IVP}_{\tilde{U}}\left(\tilde{\zeta}_{1}, \tilde{\zeta}_{2}\right)=([0,0],[1,1]),
$$

and therefore it is

$$
\operatorname{IVP}_{\tilde{U}}\left(\operatorname{IVP}_{\tilde{U}}\left(\tilde{\zeta}_{1}, \tilde{\zeta}_{2}\right), \tilde{\zeta}_{3}\right)=([0,0],[1,1]) .
$$

On the other hand, it is:

$$
\operatorname{IVP}_{\tilde{U}}\left(\tilde{\zeta}_{1}, \operatorname{IVP}_{\tilde{U}}\left(\tilde{\zeta}_{2}, \tilde{\zeta}_{3}\right)\right)=([0,0],[1,1]) .
$$

The proofs when $\tilde{\zeta}_{2}$ or $\tilde{\zeta}_{3}$ are $([0,0],[1,1])$ are the same.

$3)$ None of the IVTFs is $([1,1],[0,0])$ or $([0,0],[1,1])$. In this case, it is

$$
\operatorname{IVP}_{\tilde{U}}\left(\tilde{\zeta}_{1}, \tilde{\zeta}_{2}\right)=\left(\tilde{U}\left(\tilde{t}_{1}, \tilde{t}_{2}\right), \tilde{U}\left(\tilde{t}_{1}, \tilde{d}_{2}\right)\right),
$$

and therefore

$\operatorname{IVP}_{\tilde{U}}\left(\operatorname{IVP}_{\tilde{U}}\left(\tilde{\zeta}_{1}, \tilde{\zeta}_{2}\right), \tilde{\zeta}_{3}\right)=\left(\tilde{U}\left(\tilde{U}\left(\tilde{t}_{1}, \tilde{t}_{2}\right), \tilde{t_{3}}\right), \tilde{U}\left(\tilde{U}\left(\tilde{t}_{1}, \tilde{t}_{2}\right), \tilde{d}_{3}\right)\right)$.

Associativity of operator $\tilde{U}$ implies

$$
\begin{aligned}
\left(\tilde{U}\left(\tilde{U}\left(\tilde{t}_{1}, \tilde{t}_{2}\right), \tilde{t_{3}}\right), \tilde{U}\left(\tilde{U}\left(\tilde{t}_{1}, \tilde{t}_{2}\right), \tilde{d}_{3}\right)\right)= \\
\left(\tilde{U}\left(\tilde{t}_{1}, \tilde{U}\left(\tilde{t}_{2}, \tilde{t_{3}}\right), \tilde{U}\left(\tilde{t}_{1}, \tilde{U}\left(\tilde{t}_{2}, \tilde{d}_{3}\right)\right)\right) .\right.
\end{aligned}
$$

Given that

$$
\operatorname{IVP}_{\tilde{U}}\left(\tilde{\zeta}_{2}, \tilde{\zeta}_{3}\right)=\left(\tilde{U}\left(\tilde{t}_{2}, \tilde{t}_{3}\right), \tilde{U}\left(\tilde{t}_{2}, \tilde{d}_{3}\right)\right),
$$

it is obvious that

$$
\operatorname{IVP}_{\tilde{U}}\left(\operatorname{IVP}_{\tilde{U}}\left(\tilde{\zeta}_{1}, \tilde{\zeta}_{2}\right), \tilde{\zeta}_{3}\right)=\operatorname{IVP}_{\tilde{U}}\left(\tilde{\zeta}_{1}, \operatorname{IVP}_{\tilde{U}}\left(\tilde{\zeta}_{2}, \tilde{\zeta}_{3}\right)\right) .
$$

Thus, case 3 of the $\operatorname{IVP}_{\tilde{U}}$ for three IVTFs becomes:

$$
\operatorname{IVP}_{\tilde{U}}\left(\tilde{\zeta}_{1}, \tilde{\zeta}_{2}, \tilde{\zeta}_{3}\right)=\left(\tilde{U}\left(\tilde{t}_{1}, \tilde{t}_{2}, \tilde{t}_{3}\right), \tilde{U}\left(\tilde{t}_{1}, \tilde{t}_{2}, \tilde{d}_{3}\right)\right)
$$

For the cross-ratio uninorm $U$ given in (2),

$$
U\left(t_{1}^{-}, t_{2}^{-}, t_{3}^{-}\right)=\frac{t_{1}^{-} t_{2}^{-} t_{3}^{-}}{t_{1}^{-} t_{2}^{-} t_{3}^{-}+\left(1-t_{1}^{-}\right)\left(1-t_{2}^{-}\right)\left(1-t_{3}^{-}\right)},
$$

Similar expressions are obtained for $U\left(t_{1}^{+}, t_{2}^{+}, t_{3}^{+}\right)$, $U\left(t_{1}^{-}, t_{2}^{-}, d_{3}^{-}\right)$and $U\left(t_{1}^{+}, t_{2}^{+}, d_{3}^{+}\right)$.

Proposition 2. Let $\tilde{\zeta}_{i}=\left(\tilde{t}_{i}, \tilde{d}_{i}\right)(i=1,2 \ldots, n)$ be $n$ IVTFs. Then IVP $\tilde{U}_{\tilde{U}}$ is applied as follows:

1) If $\exists i$ such that $\tilde{\zeta}_{i}=([0,0],[1,1])$, then

$$
\operatorname{IVP}_{\tilde{U}}\left(\tilde{\zeta}_{1}, \tilde{\zeta}_{2} \ldots, \tilde{\zeta}_{n}\right)=([0,0],[1,1]) .
$$

2) For each $i$ such that $\tilde{\zeta}_{i}=([1,1],[0,0])$

$$
I V P_{\tilde{U}}\left(\tilde{\zeta}_{1}, \tilde{\zeta}_{2} \ldots \tilde{\zeta}_{n}\right)=I V P_{\tilde{U}}\left(\tilde{\zeta}_{1}, \ldots, \tilde{\zeta}_{i-1}, \tilde{\zeta}_{i+1}, \ldots, \tilde{\zeta}_{n}\right) .
$$

3) Otherwise:

$$
\begin{aligned}
& \quad \operatorname{IVP}_{\tilde{U}}\left(\tilde{\zeta}_{1}, \tilde{\zeta}_{2}, \ldots, \tilde{\zeta}_{n}\right)= \\
& \quad\left(\tilde{U}\left(\tilde{t}_{1}, \tilde{t}_{2}, \ldots, \tilde{t}_{n}\right), \tilde{U}\left(\tilde{t}_{1}, \tilde{t}_{2}, \ldots, \tilde{t}_{n-1}, \tilde{d}_{n}\right)\right) .
\end{aligned}
$$

Proof. It is easy to prove by induction and it is omitted. 


\section{Trust propagation principle in a connected network of direct trust}

Fig.1 shows a connected network of five nodes (experts) with direct trust. In Fig.1, ' $\rightarrow$ ' from expert $E_{1}$ to node $E_{2}$, for example, indicates a direct trust relationship from expert $E_{1}$ towards expert $E_{2}$. Let $\tilde{\zeta}_{p q}$ represents the IVTF of expert $E_{p}$ towards expert $E_{q}$, and $T_{L}=\left(\tilde{\zeta}_{p q}\right)_{l \times l},(p, q=1,2 \ldots l \wedge p \neq$ $q$ ) represents the trust matrix of a directed trust network of a group of experts. For two experts $E_{p}$ and $E_{q}$ with no direct

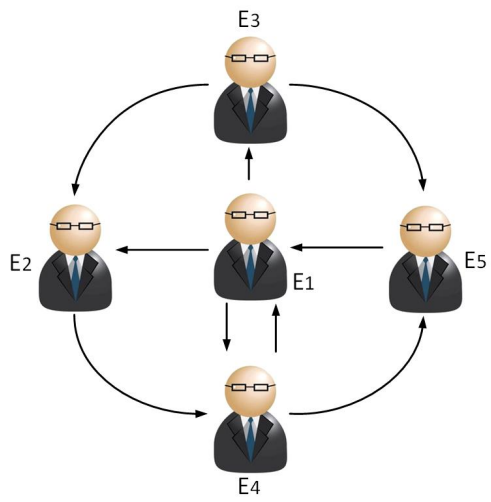

Fig. 1: Trust Network

trust relationship from $E_{p}$ towards $E_{q}$, the IVTF value $\tilde{\zeta}_{p q}$ in $T_{L}$ is unknown, as shown by the $T_{L}$ given in (22) (Section V). In a connected network, every expert can be reached from any other experts when a direct link does not exist. There could be, though, various indirect trust paths, through intermediate experts with direct links, to reach $E_{q}$ from $E_{p}$, and therefore IVTF can be propagated from $E_{p}$ towards $E_{q}$. The following propagation principle of indirect IVTF $s$ from $E_{p}$ towards $E_{q}$ is applied herein [16]: the shortest path takes precedence; if more than one path exist with shortest distance, then the average IVTF propagated in each shortest path is computed. For example, there is no direct trust relationship from $E_{3}$ towards $E_{1}$, and therefore the element $\tilde{\zeta}_{31}$ in the $T_{L}$ given in (22) is unknown. However, there are three trust propagation paths from $E_{3}$ to $E_{1}: E_{3} \rightarrow E_{5} \rightarrow E_{1} ; E_{3} \rightarrow E_{2} \rightarrow E_{4} \rightarrow E_{1}$; and $E_{3} \rightarrow E_{2} \rightarrow E_{4} \rightarrow E_{5} \rightarrow E_{1}$. Applying the trust propagation principle, we would estimate the value of $\tilde{\zeta}_{31}$ by propagating trust via the shortest path using the intermediate expert $E_{5}$. Therefore, the estimated value of $\tilde{\zeta}_{31}$ would

$$
\begin{aligned}
\tilde{\zeta}_{31}^{\prime}=\tilde{\zeta}_{31}^{5} & =\operatorname{IVP}_{\tilde{U}}\left(\tilde{\zeta}_{35}, \tilde{\zeta}_{51}\right) \\
& =\operatorname{IVP}_{\tilde{U}}(([0.6,0.8],[0.2,0.3]),([0.5,0.8],[0.2,0.4])) \\
& =(\tilde{U}([0.6,0.8],[0.5,0.8]), \tilde{U}([0.6,0.8],[0.2,0.4])) \\
& =([U(0.6,0.5), U(0.8,0.8)],[U(0.6,0.2), U(0.8,0.4)]) \\
& =([0.60,0.94],[0.27,0.73]) .
\end{aligned}
$$

There are, however, two paths of same (minimum) length from $E_{1}$ to $E_{5}$, via the intermediate expert $E_{3}$ and via the intermediate expert $E_{4}$, respectively. In this case, the estimated value of $\tilde{\zeta}_{15}$ would be:

$$
\tilde{\zeta}_{15}^{\prime}=\frac{\tilde{\zeta}_{15}^{3}+\tilde{\zeta}_{15}^{4}}{2}=([0.55,0.95],[0.13,0.59]) \text {. }
$$

Where,

$$
\begin{aligned}
\tilde{\zeta}_{15}^{3} & =\operatorname{IVP}_{\tilde{U}}\left(\tilde{\zeta}_{13}, \tilde{\zeta}_{35}\right) \\
& =\operatorname{IVP}_{\tilde{U}}(([0.5,0.9],[0.3,0.5]),([0.6,0.8],[0.2,0.3])) \\
& =([0.60,0.97],[0.20,0.79]) . \\
\tilde{\zeta}_{15}^{4} & =\operatorname{IVP}_{\tilde{U}}\left(\tilde{\zeta}_{14}, \tilde{\zeta}_{45}\right) \\
& =\operatorname{IVP}_{\tilde{U}}(([0.4,0.6],[0.5,0.7]),([0.6,0.9],[0.1,0.3])) \\
& =([0.50,0.93],[0.07,0.39]) .
\end{aligned}
$$

By this way, the unknown elements of the $T_{L}$ between a group of experts in a connected network of direct trust can be estimated, and the complete trust matrix $T_{L}^{\prime}$ derived, which for the example used herein is given in (23) (Section V).

\section{A TWOFOLD PERSONALIZED FEEDBACK MECHANISM FOR GDM}

\section{A. Identification of consensus inconsistency}

Three consensus levels are a powerful tool for identifying inconsistency in a GDM problem. Let $E=\left\{e_{1}, e_{2} \ldots e_{l}\right\}$ be a set of 1 experts, $A=\left\{A_{1}, A_{2} \ldots A_{m}\right\}$ be a set of $\mathrm{m}$ alternatives and $C=\left\{c_{1}, c_{2} \ldots c_{n}\right\}$ be a set of $\mathrm{n}$ criterions. Given the experts set of decision matrices $\left\{\tilde{R}^{h}=\left(\tilde{r}_{i j}^{h}\right)_{m \times n}, \tilde{r}_{i j}^{h}=\right.$ $\left.\left(\tilde{t}_{i j}^{h}, \tilde{d}_{i j}^{h}\right) ; h=1, \ldots, l.\right\}$, where $\tilde{r}_{i j}^{h}$ is an $I V T F$, which represents the $e_{h}$ judgment of how well alternative $A_{i}$ fulfill $c_{j}$. Then, consensus can be measured with respect to any of the elements of the decision matrix (element level), with respect to any of its rows of elements (alternative level); or with respect to all its elements (decision matrix level):

a) Consensus with respect to a decision matrix element: The value

$$
\begin{aligned}
& \operatorname{CDE}_{i j}\left(\tilde{r}^{h}, \tilde{r}^{k}\right) \\
& =1-\frac{\left|t_{i j}^{h-}-t_{i j}^{k-}\right|+\left|t_{i j}^{h+}-t_{i j}^{k+}\right|+\left|d_{i j}^{h-}-d_{i j}^{k-}\right|+\left|d_{i j}^{h+}-d_{i j}^{k+}\right|}{4}
\end{aligned}
$$

measures closeness, and therefore similarity or agreement between the preferences of experts $e_{h}$ and $e_{k}$ on alternative $A_{i}$ with respect to criterion $c_{j}$, which can be tracked to[17].The value

$$
A \mathrm{CDE}_{i j}^{h}=\frac{1}{l-1} \sum_{h \neq k, k=1}^{l} \operatorname{CDE}_{i j}\left(\tilde{r}^{h}, \tilde{r}^{k}\right)
$$

measures the average degree of similarity or consensus of expert $e_{h}$ with the rest of experts in the group on alternative $A_{i}$ with respect to criterion $c_{j}$.

b) Consensus with respect to an alternative: The value

$$
\operatorname{ACDA}_{i}^{h}=\frac{1}{n} \sum_{j=1}^{n} A \operatorname{CDE}_{i j}^{h}
$$

measures the average degree of similarity or consensus of expert $e_{h}$ with the rest of experts in the group on alternative $A_{i}$.

c) Consensus degree on the decision matrix: The value

$$
\operatorname{ACDD}^{h}=\frac{1}{m} \sum_{i=1}^{m} \operatorname{ACDA}_{i}^{h}
$$

measures the average degree of similarity or consensus of expert $e_{h}$ with the rest of experts in the group. 
Complete consensus is obtained only when all experts have same preferences, which is not expected and may not be required in practice [45], where a sufficiently high level of consensus $\gamma$ would be sufficient for the group of experts to 'agree on disagree' [46]. Without loss of generality, this article sets the threshold value of consensus at $\gamma=0.8$, which means that if an expert has $\operatorname{ACDD}^{h}$ below $\gamma$, then such expert is labelled as 'inconsistent'.

d) Inconsistent experts identification process refinement: Once the set of inconsistent experts are identified:

$$
\mathrm{EXPCH}=\left\{h \mid \mathrm{ACDD}^{h}<\gamma\right\},
$$

their corresponding 'inconsistent' alternatives are identified

$$
\mathrm{ALT}=\left\{(h, i) \mid h \in \mathrm{EXPCH} \wedge \mathrm{ACDA}_{i}^{h}<\gamma\right\},
$$

which is refined into find the their 'inconsistent' preferences on specific elements of their decision matrices

$$
\mathrm{APS}=\left\{(h, i, j) \mid(h, i) \in \mathrm{ALT} \wedge A \mathrm{CDE}_{i j}^{h}<\gamma\right\}
$$

\section{B. Traditional feedback mechanism}

Feedback mechanism within a consensus reaching process refers to the procedure of producing advice or recommendations to inconsistent experts to support them in reaching the threshold value of consensus agreeable to the group. Advice can be presented as follows: "Revise your preference value on alternative $A_{i}$ under criterion $c_{j}$ from $\tilde{r}_{i j}^{h}=\left(\tilde{t}_{i j}^{h}, \tilde{d}_{i j}^{h}\right)$ to $r \tilde{r}_{i j}^{h}=\left(r \tilde{t}_{i j}^{h}, r \tilde{d}_{i j}^{h}\right)$ ". Reaching the threshold value of consensus requires that inconsistent values are revised and considered to be replaced by adjustment advice closer to the corresponding 'group values', i.e.

$$
\begin{aligned}
r \tilde{r}_{i j}^{h} & =(1-\delta) \cdot \tilde{r}_{i j}^{h}+\delta \cdot \tilde{\tilde{r}}_{i j}^{h} \\
& =\left((1-\delta) \cdot \tilde{t}_{i j}^{h}+\delta \cdot \tilde{\tilde{t}}_{i j}^{h},(1-\delta) \cdot \tilde{d}_{i j}^{h}+\delta \cdot \overline{\tilde{d}}_{i j}^{h}\right),
\end{aligned}
$$

where $\delta \in[0,1]$ is a feedback coefficient that controls the extent of the adjustment change or cost for the inconsistent expert; $\overline{\tilde{r}}_{i j}^{h}=\frac{1}{l} \sum_{k=1}^{l} \tilde{r}_{i j}^{k}=\left(\frac{1}{l} \sum_{k=1}^{l} \tilde{t}_{i j}^{k}, \frac{1}{l} \sum_{k=1}^{l} \tilde{d}_{i j}^{k}\right)$.

There are two main drawbacks associated to this 'traditional' feedback mechanism [35], [37]: (1) the 'group value' used above is the group average value. This may not be the best to use in a trust social network because it neglects the individual trust relationships, which is a key in accepting advice or recommendations willingly [12], [17], [37]; (2) the feedback coefficient $\delta$ is fixed and common to all 'inconsistent' experts; this means that different states of inconsistency will be treated equally, i.e. an inconsistent expert will be subjected to the same adjustment cost regardless of how close they are from the threshold value of consensus.

The proposed twofold personalized feedback mechanism herein will overcome these two drawbacks. On the one hand, the average operator used in the traditional feedback process is repacked by a more general operator able to implement the trust relationship between a group of experts in an appropriate way. On the other hand, personalized adoption coefficients for inconsistent experts that minimise the feedback adjustment cost are proposed instead. To do that, in the next section, a twofold personalized feedback mechanism is proposed to determine personalized adoption coefficients for them with the goal of minimizing feedback cost.

\section{Trust based personalized feedback mechanism}

The implementation of the trust relationship between the group of experts in the feedback mechanism results in the provision of trust based personalized feedback advice. The below interval-value trust score induced ordered weighted average $(O W A)$ operator weight assignment process generates group opinions, which are used in the computation of personalized feedback adjustment advice for inconsistent experts.

1) As per Section III-C, let $T_{L}=\left(\tilde{\zeta}_{p q}\right)_{l \times l},(p, q=$ $1,2 \ldots l \wedge p \neq q)$ be the completed trust matrix of a connected network of direct trust between a group of experts, with $\tilde{\zeta}_{p q}$ representing the IVTF of expert $E_{p}$ towards expert $E_{q}$.

2) If $h \in \mathrm{EXPCH}$, then row $h$ of matrix $T_{L},\left\{\tilde{\zeta}_{h k} \mid k \neq\right.$ $h\}$, is arranged in descending order, denoted as follows: $\tilde{\zeta}_{\sigma_{h}(l-1)} \preceq \tilde{\zeta}_{\sigma_{h}(l-2)} \preceq \ldots \preceq \tilde{\zeta}_{\sigma_{h}(1)}$. The below total ordering on the set of IVTFs, based on the trust score $T S_{\tilde{\zeta}}=\left(t^{-}+t^{+}-d^{-}-d^{+}+2\right) / 4$ and knowledge degree $K D_{\tilde{\zeta}}=\left|1-\left(t^{-}+t^{+}+d^{-}+d^{+}\right) / 2\right|$, was proposed in [17]: $\tilde{\zeta}_{i} \prec \tilde{\zeta}_{j} \Leftrightarrow\left[T S_{\tilde{\zeta}_{i}}<T S_{\tilde{\zeta}_{j}}\right] \vee\left[T S_{\tilde{\zeta}_{i}}=T S_{\tilde{\zeta}_{j}} \wedge\right.$ $\left.K D_{\tilde{\zeta}_{i}}>K D_{\tilde{\zeta}_{j}}\right]$.

3) Denoting $H(\sigma(s))=\sum_{u=1}^{s} T S_{\zeta_{\sigma_{h}(u)}}$, it is the sum of the trust scores of $\tilde{\zeta}$ in the top $s$. The following personalized weighting vector is allocated to expert $E_{h}:\left\{\tilde{w}^{\sigma_{h}(s)} \mid s=\right.$ $1, \ldots, l-1\}$ where

$$
\tilde{w}^{\sigma_{h}(s)}=Q\left(\frac{H(\sigma(s))}{H(\sigma(l-1))}\right)-Q\left(\frac{H(\sigma(s-1))}{H(\sigma(l-1))}\right)
$$

with $Q(x)=\sqrt{x}$ representing the concept of 'majority' as per the group consensus reaching model described in [37]. Thus, for inconsistent experts $E_{h}(h \in \mathrm{EXPCH})$, their personal trust based weighting vectors towards the other experts in the group, which we denote as $\tilde{W}_{h \rightarrow k}=$ $\left(\tilde{w}_{h \rightarrow 1}, \ldots, \tilde{w}_{h \rightarrow h-1}, \tilde{w}_{h \rightarrow h+1}, \ldots, \tilde{w}_{h \rightarrow l}\right)$, are obtained and their corresponding personalized group values are generated.

4) The personalized group value for the inconsistent expert $E_{h}$ on the element $\left(A_{i}, c_{j}\right)$, i.e $(h, i, j) \in \mathrm{APS}$, is

$$
\tilde{r}_{i j}^{T h}=\sum_{k \neq h, k=1}^{l} \tilde{w}_{h \rightarrow k} \cdot \tilde{r}_{i j}^{k} .
$$

5) The personalized feedback advice for each $(h, i, j) \in$ APS is computed as follows

$$
r \tilde{r}_{i j}^{h}=\left(1-\delta^{h}\right) \cdot \tilde{r}_{i j}^{h}+\delta^{h} \cdot \tilde{r}_{i j}^{T h},
$$

where $\delta^{h}$ represent the personal adoption coefficient for the inconsistent expert $E_{h}$, whose computation is described in the next section.

\section{Personalized adoption coefficient based on minimum cost}

There are two expectations for the value of $\delta^{h}$ : one is to make the inconsistent expert's consensus degree on the decision matrix, $\mathrm{ACDD}^{h}$, to reach the threshold of consensus 
after feedback adjustment; the other one is that the adjusting costs should be optimized.

Adjustment cost is measured as the difference between an expert's inconsistent preference value, $\tilde{r}_{i j}^{h}$ such that $(h, i, j) \in$ APS, and the corresponding personalized feedback advice that replaces it, $r \tilde{r}_{i j}^{h}$,

$$
c_{i j}^{h}=\left|\tilde{r}_{i j}^{h}-r \tilde{r}_{i j}^{h}\right|=\delta^{h}\left|\tilde{r}_{i j}^{h}-\tilde{r}_{i j}^{T h}\right|
$$

Solving the below twofold personalized feedback model for the inconsistent experts will result in their personalized adoption coefficients with minimum cost to reach the threshold value of consensus $\gamma$ :

$$
\begin{cases}\min : & \sum_{(h, i, j) \in \mathrm{APS}} \delta^{h}\left|\tilde{r}_{i j}^{h}-\tilde{r}_{i j}^{T h}\right| \\
\text { s.t. } & \left\{\begin{array}{l}
\tilde{r}_{i j}^{T h}=\sum_{k \neq h, k=1}^{l} \tilde{w}_{h \rightarrow k} \cdot \tilde{r}_{i j}^{k} \\
\overline{\mathrm{ACDD}}^{k} \geq \gamma(h \in \mathrm{EXPCH}) \\
\overline{\mathrm{ACDD}}^{k} \geq \gamma(k \neq h) \\
0 \leq \delta^{h} \leq 1
\end{array}\right.\end{cases}
$$

where the targeted consensus degrees, $\overline{\mathrm{ACDD}}^{h}$, at the next round of interaction for inconsistent experts $E_{h}$ reach the threshold value of consensus, while keeping the rest of experts consensus degrees, $\overline{\mathrm{ACDD}}^{k}$, as they were before the feedback process, i.e. greater or equal to the threshold value of consensus.

\section{E. Selection process for GDM}

Once all experts' consensus degree on the decision matrix reach the threshold of consensus, $\overline{\mathrm{ACDD}}^{h} \geq \gamma(\forall h)$, the individual decision matrices are aggregated using an induced OWA operator with weights, $\left(w_{1}, \ldots, w_{l}\right)$, derived using Exp. (16) with the set of valued $\left\{\overline{\mathrm{ACDD}}^{1}, \ldots, \overline{\mathrm{ACDD}}^{l}\right\}$ arranged in descending order. Assuming that the set of criteria $\left\{C_{1}, \ldots, C_{n}\right\}$ has associated a set of importance weights $\left\{w_{C_{1}}, \ldots, w_{C_{n}}\right\}$, then each alternative $\left\{A_{i} ; i=1, \ldots, m\right\}$ will be associated the following IVTF:

$$
\bar{R}_{i}=\sum_{j=1}^{n} w_{C_{j}}\left(\sum_{h=1}^{l} w_{h} \tilde{r}_{i j}^{h}\right)
$$

A total ordering of the alternatives is possible by applying the total ordering on the set of IVTFs used in Section IV-C.

\section{F. Twofold personalized feedback algorithm}

The proposed twofold personalized feedback mechanism based on a connected interval trust network for consensus in GDM algorithmic description is presented below:

\section{ILluStRATIVE EXAMPLE}

A group of five experts $\left\{E_{1}, E_{2}, E_{3}, E_{4}, E_{5}\right\}$ has to decide on a garment factory site for a clothing firm from given four alternative sites $\left\{A_{1}, A_{2}, A_{3}, A_{4}\right\}$. The alternative sites are evaluated using four criteria: $c_{1}$ : infrastructure; $c_{2}$ : climate; $c_{3}$ : cost; and $c_{4}$ : environmental protection. They have original trust relationship network as per Fig.1 with incomplete trust

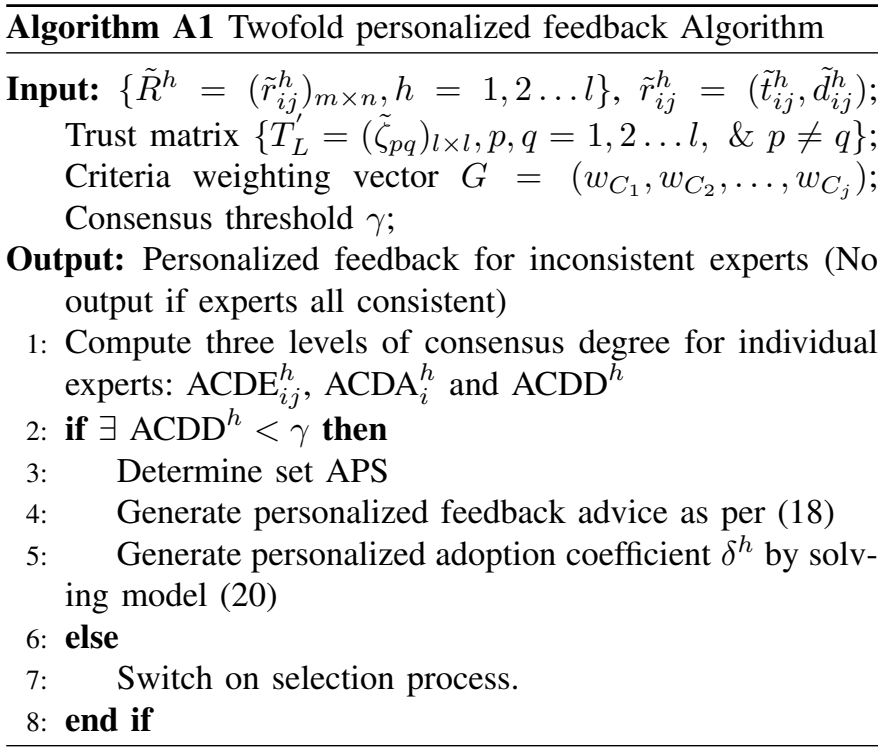

relationship matrix $T_{L}$, which is estimated and completed in matrix $T_{L}^{\prime}$. The five experts original decision matrices $\tilde{R}^{1}$, $\tilde{R}^{2}, \tilde{R}^{3}, \tilde{R}^{4}, \tilde{R}^{5}$ are also provided below. Group threshold of consensus is set at $\gamma=0.8$

a) Identification of consensus inconsistency: The experts' consensus degrees on the decision matrix are $\mathrm{ACDD}^{1}=0.8195 ; \mathrm{ACDD}^{2}=0.8051 ; \mathrm{ACDD}^{3}=$ 0.8168; $\quad \mathrm{ACDD}^{4}=0.7699 ; \quad \mathrm{ACDD}^{5}=0.7195$, which means that experts $E_{4}$ and $E_{5}$ are inconsistent. The inconsistent experts identification process refinement yields APS = $\{(4,2,3),(4,4,1),(4,4,3),(5,1,1),(5,1,4),(5,2,3),(5,2,4)\}$.

b) Allocation of weights to inconsistent experts: Arranging in descending order rows 4 and 5 of matrix $T_{L}^{\prime}$, respectively, and applying expression (16), inconsistent experts $E_{4}$ and $E_{5}$ are allocated the following vectors of trust weights towards the rest of experts in the group:

- $E_{4}$ vector of trust weights towards $\left(E_{1}, E_{2}, E_{3}, E_{5}\right)$ is $\tilde{W}_{4 \rightarrow k}=(0.16,0.52,0.11,0.21)$.

- $E_{5}$ vector of trust weights towards $\left(E_{1}, E_{2}, E_{3}, E_{4}\right)$ is $\tilde{W}_{5 \rightarrow k}=(0.21,0.55,0.14,0.10)$.

c) Personalized adoption coefficient by twofold personalized feedback mechanism: The optimisation model (20) becomes 


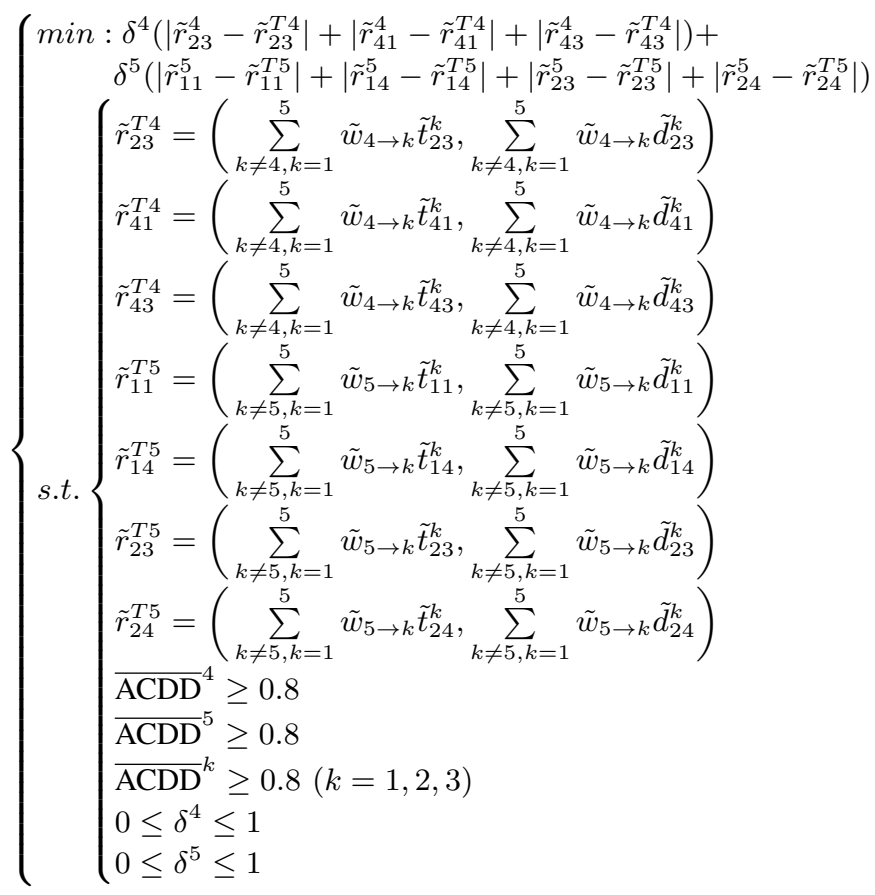

The solution of this optimisation model results in the following personalized adoption coefficients for $E_{4}$ and $E_{5}$ : $\delta^{4}=0.178$ and $\delta^{5}=0.577$.An example of one personalized feedback advice for $E_{4}$ and $E_{5}$ are given below:

$E_{4}$ : "Revise your preference value on alternative $A_{2}$ under criterion $c_{3}$ from $([0.1,0.2],[0.3,0.4])$ to $([0.187,0.294],[0.354,0.463])$;

$E_{5}$ : "Revise your preference value on alternative $A_{1}$ under criterion $c_{1}$ from $([0.1,0.2],[0.8,0.9])$ to $([0.446,0.588],[0.416,0.516])$;

The twofold personalized feedback model results in the below experts' consensus values: $\overline{\mathrm{ACDD}}^{1}=0.8425 ; \overline{\mathrm{ACDD}}^{2}=$ $0.8268 ; \overline{\mathrm{ACDD}}^{3}=0.8394 ; \overline{\mathrm{ACDD}}^{4}=0.8000 ; \overline{\mathrm{ACDD}}^{5}=$ 0.8000 .

d) Selection Process: Applying Exp. (16) the experts weights are $w_{1}=0.453 ; w_{2}=0.142 ; w_{3}=0.187 ; w_{4}=$ $0.109 ; w_{5}=0.109$, with when implemented with the following criteria weighing vector $G=(0.1,0.25,0.4,0.25)$ results in

$$
\bar{R}=\left[\begin{array}{ll}
A_{1} & ([0.548,0.752],[0.259,0.381]) \\
A_{2} & ([0.302,0.459],[0.427,0.582]) \\
A_{3} & ([0.387,0.640],[0.315,0.506]) \\
A_{4} & ([0.305,0.602],[0.328,0.505])
\end{array}\right]
$$

The final ranking of alternatives is: $A_{1} \succ A_{3} \succ A_{4} \succ A_{2}$.

\section{COMPARISON AND DISCUSSION}

A comparative analysis between the traditional feedback model, the non-personalised feedback model and the proposed twofold personalized feedback model is provided, which is shown in TableI, TableII, and Figs.2-3.

Table I summarises the main operation mechanisms for the traditional, non-personalized and twofold personalized feedback models with respect to: (1) the feedback advice construction; (2) the derivation of the adoption coefficients; and (3) the objective function to achieve. While the traditional and and non-personalized feedback models base their feedback advices on using the same group opinion average to generate feedback advices for inconsistent experts, the proposed twofold personalized feedback generates advices based on personalised group opinion weighted average based on each individual inconsistent expert's trust relationship. The main effect is shown in Fig. 3: Fig. 3 (a) and Fig. 3 (b) shows that the inconsistent experts are somehow caught up in the 'group thinking', as their final positions are closer to the group opinion than in the case of the proposed twofold personalized feedback, which as shown in Fig. 3 (c), where the approach to the group opinion by the inconsistent experts is the minimum required achieving the threshold value of consensus and, therefore it is possible to achieve group diversity by keeping their personality traits as much as possible.

Table II exhibits the detailed results of the three feedback mechanisms in terms of Recommendation advice, Adoption coefficients, and Objective functions, also shows their respective feedback effects from the sum of feedback coefficients and the total cost of adjustment with the same target threshold $(\gamma=0.8)$. In the respect of feedback advice, $\bar{E}$ is the average group's original opinions and the representation of 'group thinking', which is applied in the operation of model (a) and model (b); $\hat{E}_{4}$ and $\hat{E}_{5}$ are personalized group opinions generated based on individual trust relationships, which is a unique opinion for generating feedback advice in the model proposed in this paper. For the adoption coefficient, model (a) randomly determines the value that meets its objective function, and here the value was found to be $\delta^{4}=\delta^{5}=0.7$ (accurate to one decimal place); model (b) and model (c) are the calculation results with their objective function and conditions. As a result, each model can achieve its pre-set goals $(\gamma=0.8)$, but the final feedback status and adjust cost are different. Specifically, the proposed twofold personalized feedback model achieved the minimum values. The nonpersonalized feedback model is superior to the traditional feedback model in that the changes to implement lead to a lower total adjustment cost, which means that minimizing cost contributes towards the keeping of the personality traits. This is supplemented by the twofold personalized feedback model with the implementation of personal trust relationships. Indeed, the non-personalized feedback model produces values for the adoption coefficients and total adjustment cost that are between the maximum values obtained with the traditional feedback model and the minimum values obtained with the twofold personalized feedback model.To further prove this, a visual simulation result is shown in Fig.2. The results show that in the case of different target thresholds (Case 1: $\gamma=$ $0.75 ; E X P C H=\{5\} ;$ Case $2: \gamma=0.8 ; E X P C H=\{5,4\}$; Case3: $\gamma=0.81 ; E X P C H=\{5,4,2\})$, twofold personalized feedback model always achieved the minimum total cost. Noticed that the performance of the three feedback models is not much different in case 1 , but the adjustment cost of the twofold personalized feedback model in case 2 and case 3 is significantly lower than the other two models, which means that the twofold personalized feedback mechanism is more effective in dealing with the feedback with multiple inconsistent experts. 


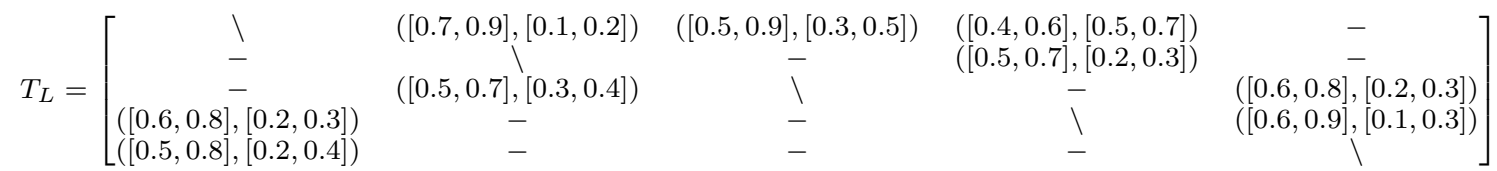

$$
\begin{aligned}
& T_{L}^{\prime}=\left[\begin{array}{c}
([0.60,0.90],[0.20,0.50]) \\
([0.60,0.94],[0.27,0.73]) \\
([0.6,0.8],[0.2,0.3]) \\
([0.5,0.8],[0.2,0.4])
\end{array}\right. \\
& ([0.7,0.9],[0.1,0.2]) \\
& ([0.5,0.7],[0.3,0.4]) \\
& \begin{array}{c}
([0.78,0.97],[0.14,0.50]) \\
([0.70,0.97],[0.10,0.50])
\end{array}
\end{aligned}
$$

$$
\begin{aligned}
& \tilde{R}^{1}=\left[\begin{array}{ccccc} 
& c_{1} & c_{2} & c_{3} & c_{4} \\
A_{1} & ([0.5,0.9],[0.2,0.3]) & ([0.7,0.9],[0.4,0.5]) & ([0.5,0.7],[0.3,0.4]) & ([0.4,0.7],[0.1,0.3]) \\
A_{2} & ([0.5,0.6],[0.1,0.2]) & ([0.5,0.7],[0.2,0.5]) & ([0.1,0.3],[0.8,0.9]) & ([0.1,0.2],[0.2,0.3]) \\
A_{3} & ([0.6,0.7],[0.1,0.2]) & ([0.5,0.8],[0.1,0.3]) & ([0.3,0.6],[0.4,0.5]) & ([0.2,0.4],[0.3,0.6]) \\
A_{4} & ([0.4,0.6],[0.2,0.3]) & ([0.3,0.5],[0.3,0.6]) & ([0.1,0.8],[0.2,0.3]) & ([0.5,0.8],[0.3,0.5])
\end{array}\right] \\
& \tilde{R}^{2}=\left[\begin{array}{ccccc} 
& c_{1} & c_{2} & c_{3} & c_{4} \\
A_{1} & ([0.8,0.9],[0.1,0.2]) & ([0.4,0.7],[0.2,0.3]) & ([0.5,0.6],[0.2,0.4]) & ([0.6,0.8],[0.1,0.2]) \\
A_{2} & ([0.8,0.9],[0.2,0.4]) & ([0.3,0.4],[0.6,0.8]) & ([0.8,0.9],[0.7,0.9]) & ([0.1,0.2],[0.1,0.2]) \\
A_{3} & ([0.6,0.8],[0.1,0.2]) & ([0.3,0.6],[0.4,0.5]) & ([0.4,0.6],[0.3,0.6]) & ([0.3,0.6],[0.4,0.5]) \\
A_{4} & ([0.5,0.6],[0.2,0.3]) & ([0.1,0.3],[0.5,0.7]) & ([0.5,0.9],[0.3,0.5]) & ([0.1,0.3],[0.5,0.7])
\end{array}\right] \\
& \tilde{R}^{3}=\left[\begin{array}{ccccc} 
& c_{1} & c_{2} & c_{3} & c_{4} \\
A_{1} & ([0.6,0.7],[0.2,0.3]) & ([0.7,0.8],[0.2,0.3]) & ([0.4,0.7],[0.5,0.6]) & ([0.7,0.8],[0.1,0.2]) \\
A_{2} & ([0.4,0.6],[0.3,0.4]) & ([0.4,0.6],[0.5,0.8]) & ([0.1,0.2],[0.8,0.9]) & ([0.2,0.3],[0.1,0.2]) \\
A_{3} & ([0.6,0.7],[0.1,0.3]) & ([0.5,0.8],[0.3,0.4]) & ([0.5,0.8],[0.3,0.5]) & ([0.2,0.5],[0.6,0.9]) \\
A_{4} & ([0.4,0.6],[0.2,0.3]) & ([0.2,0.4],[0.5,0.6]) & ([0.3,0.4],[0.4,0.6]) & ([0.6,0.7],[0.5,0.7])
\end{array}\right] \\
& \tilde{R}^{4}=\left[\begin{array}{ccccc} 
& c_{1} & c_{2} & c_{3} & c_{4} \\
A_{1} & ([0.7,0.9],[0.1,0.2]) & ([0.7,0.9],[0.4,0.5]) & ([0.4,0.5],[0.2,0.3]) & ([0.7,0.9],[0.3,0.4]) \\
A_{2} & ([0.8,0.9],[0.1,0.2]) & ([0.2,0.5],[0.3,0.5]) & ([0.1,0.2],[0.3,0.4]) & ([0.1,0.3],[0.3,0.4]) \\
A_{3} & ([0.6,0.7],[0.4,0.5]) & ([0.3,0.4],[0.2,0.5]) & ([0.6,0.8],[0.5,0.7]) & ([0.6,0.8],[0.2,0.4]) \\
A_{4} & ([0.1,0.2],[0.8,0.9]) & ([0.2,0.5],[0.6,0.7]) & ([0.1,0.2],[0.7,0.8]) & ([0.3,0.4],[0.3,0.5])
\end{array}\right] \\
& \tilde{R}^{5}=\left[\begin{array}{ccccc} 
& c_{1} & c_{2} & c_{3} & c_{4} \\
A_{1} & ([0.1,0.2],[0.8,0.9]) & ([0.7,0.8],[0.1,0.2]) & ([0.7,0.9],[0.3,0.5]) & ([0.1,0.2],[0.8,0.9]) \\
A_{2} & ([0.4,0.9],[0.4,0.5]) & ([0.5,0.8],[0.2,0.6]) & ([0.7,0.9],[0.1,0.2]) & ([0.8,0.9],[0.6,0.7]) \\
A_{3} & ([0.3,0.5],[0.1,0.4]) & ([0.4,0.5],[0.3,0.4]) & ([0.1,0.6],[0.5,0.9]) & ([0.6,0.8],[0.4,0.5]) \\
A_{4} & ([0.4,0.6],[0.2,0.3]) & ([0.3,0.5],[0.1,0.3]) & ([0.4,0.6],[0.1,0.4]) & ([0.6,0.8],[0.4,0.6])
\end{array}\right]
\end{aligned}
$$

TABLE I: Comparison of three models wrt adoption coefficient, feedback advice and objective function

\begin{tabular}{llll}
\hline Models & Recommendation advice & Adoption coefficient & Objective Function \\
\hline $\begin{array}{l}\text { (a) Traditional feedback } \\
\text { model[17] }\end{array}$ & Group opinion average & $\begin{array}{l}\text { Randomly generated and com- } \\
\text { mon to all inconsistent experts }\end{array}$ & $\begin{array}{l}\text { Inconsistent experts' consensus } \\
\text { value greater than or equal to } \\
\text { threshold value }\end{array}$ \\
$\begin{array}{l}\text { (b) Non-personalized } \\
\text { feedback model[23] }\end{array}$ & Group opinion average & $\begin{array}{l}\text { Determined by minimizing cost } \\
\text { objective function and common } \\
\text { to all inconsistent experts }\end{array}$ & $\begin{array}{l}\text { Inconsistent experts' consensus } \\
\text { value greater than or equal to } \\
\text { threshold value with minimum } \\
\text { cost }\end{array}$ \\
$\begin{array}{llll}\text { (c) Twofold personal- } \\
\text { ized feedback model }\end{array}$ & $\begin{array}{l}\text { Personalised group opinion } \\
\text { weighted average based on } \\
\text { individual's trust relationship }\end{array}$ & $\begin{array}{l}\text { Determined by minimizing cost } \\
\text { objective function and personal- } \\
\text { ized for each inconsistent experts }\end{array}$ & $\begin{array}{l}\text { value equal to threshold value } \\
\text { withimum cost }\end{array}$ \\
\hline
\end{tabular}


TABLE II: Compared models values wrt adoption coefficient, consensus degree and total cost (minimum value highlighted)

\begin{tabular}{|c|c|c|c|}
\hline & $\begin{array}{l}\text { (a) Traditional feedback } \\
\text { model[17] }\end{array}$ & $\begin{array}{l}\text { (b) Non-personalized feedback } \\
\text { model[23] }\end{array}$ & $\begin{array}{l}\text { (c) Twofold personalized } \\
\text { feedback model }\end{array}$ \\
\hline \multirow{4}{*}{$\begin{array}{l}\text { Recommendation } \\
\text { advice }\end{array}$} & $\bar{E}$ for $(4,2,3)$ is & $\bar{E}$ for $(4,2,3)$ is & \multirow{5}{*}{$\begin{array}{c}\hat{E}_{4} \text { for }(4,2,3) \text { is } \\
([0.59,0.72],[0.601,0.753]) \ldots \\
\hat{E}_{5} \text { for }(5,1,1) \text { is } \\
([0.699,0.872],[0.135,0.235]) \ldots \\
\delta^{4}=\mathbf{0 . 1 7 8} \\
\delta^{5}=\mathbf{0 . 5 7 7}\end{array}$} \\
\hline & $([0.36,0.5],[0.54,0.66]) \ldots$ & $([0.36,0.5],[0.54,0.66]) \ldots$ & \\
\hline & for $(5,1,1)$ is & for $(5,1,1)$ is & \\
\hline & $([0.54,0.72],[0.28,0.38]) \ldots$ & $([0.54,0.72],[0.28,0.38]) \ldots$ & \\
\hline $\begin{array}{l}\text { Adoption } \\
\text { coefficient }\end{array}$ & $\delta^{4}=\delta^{5}=0.7$ & $\delta^{4}=\delta^{5}=0.667$ & \\
\hline \multirow{2}{*}{$\begin{array}{l}\text { Objective } \\
\text { function }\end{array}$} & $\overline{\mathrm{ACDD}}^{4}=0.8218$ & $\overline{\mathrm{ACDD}}^{4}=0.8202$ & \multirow{2}{*}{$\begin{array}{c}\overline{\mathrm{ACDD}}^{4}=\mathbf{0 . 8 0 0 0} \\
\overline{\mathrm{ACDD}}^{5}=\mathbf{0 . 8 0 0 0}\end{array}$} \\
\hline & $\overline{\mathrm{ACDD}}^{5}=0.8028$ & $\overline{\mathrm{ACDD}}^{5}=\mathbf{0 . 8 0 0 0}$ & \\
\hline$\sum \delta^{h}$ & 1.4 & 1.354 & 0.755 \\
\hline Total cost & 1.939 & 1.875 & 1.540 \\
\hline
\end{tabular}

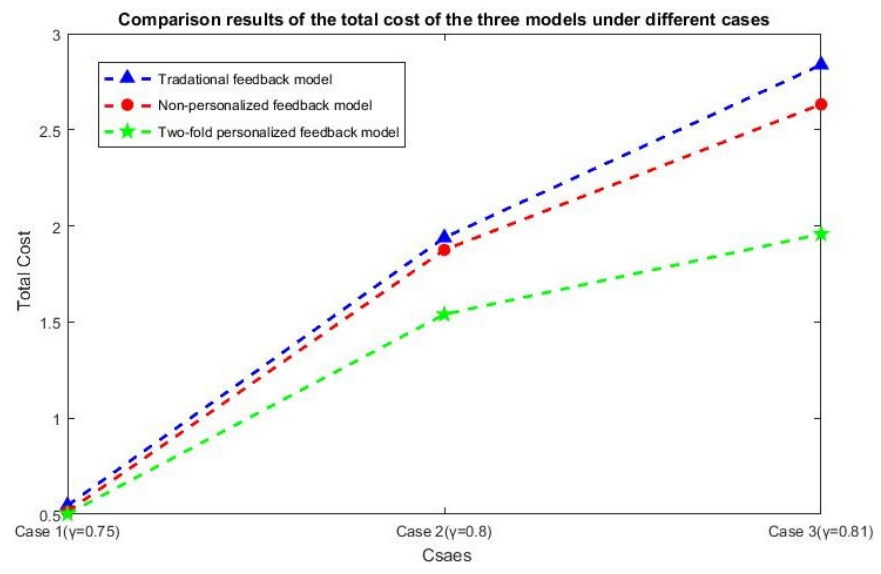

Fig. 2: Comparison results of adjustment costs of three models in three cases

In realistic cases, inconsistent experts are more willing to change their opinions closer to their most trusted experts' opinions, as visualised in Fig.3 (c) with $\hat{E}_{4}$ and $\hat{E}_{5}$. The personalized feedback mechanism focuses on keeping the 'individual personality' as much as possible while achieving as well the 'group goal' of consensus. Thus, the personalized feedback mechanism contributes to achieving agreement while maintaining group diversity with minimizing adjustment cost, which can be referred to as "achieving agreement while disagreeing". Both traditional and non-personalized feedback models put all or some of the inconsistent experts' consensus degrees above the satisfactory group threshold of consensus, and as a consequence unnecessary over-adjustments are imposed on the inconsistent experts' that are moved above the threshold of consensus.

\section{CONCLUSIONS}

This article proposed a twofold personalized feedback mechanism by a uninorm interval trust propagation operator to help inconsistent experts reach the consensus threshold value in social network group decision making (SN-GDM). The proposed model has the following features :
(1) It is based on an extension of the numerical uninorm trust propagation operators to the case of interval numbers, which enriches the field of trust propagation. The concept of trust and distrust expressed by interval numbers can better capture the uncertainty of experts' opinions in SN-GDM. The uninorm interval-valued trust propagation operator is developed to provide a new method to compute indirect trust relationship.

(2) Personalized feedbacks advices are generated for each one of the inconsistent experts based on their individual trust network. This approach is able to maintain the personality traits of individual experts, and therefore guarantee group diversity. Hence, it can resolve the 'group thinking' issue of traditional feedback mechanisms. This is indeed possible because the personalization resides in personal individual adoption coefficients for inconsistent experts obtained by minimizing adjustment cost. This is argued to be an appropriate methodology to ensure that the feedback advice are accepted by the inconsistent experts.

This article used trust as a reliable source to investigate a novel feedback mechanism for consensus reaching process in a small-scale group decision making; its essence is a 'decentralization' mechanism that can serve the consensus reached in the Blockchain. However, this research also has some shortcomings. For example, the knowledge defects of experts are ignored in trust propagation where exists multiple feasible paths. In the future, this element will be took account to relevant research. In addition, research efforts will be put into implementing this proposed methodology in a large-scale group decision making framework.

\section{ACKNOWLEDGEMENTS}

The authors would like to thank the editors and anonymous referees for their careful work and valuable comments and suggestions that have helped improve this paper substantially. This work was sponsored by National Natural Science Foundation of China (NSFC) (No. 71971135, 71571166 and 71910107002), and was supported in part by the Spanish State Research Agency under Project PID2019-103880RBI00/AEI/10.13039/501100011033. 


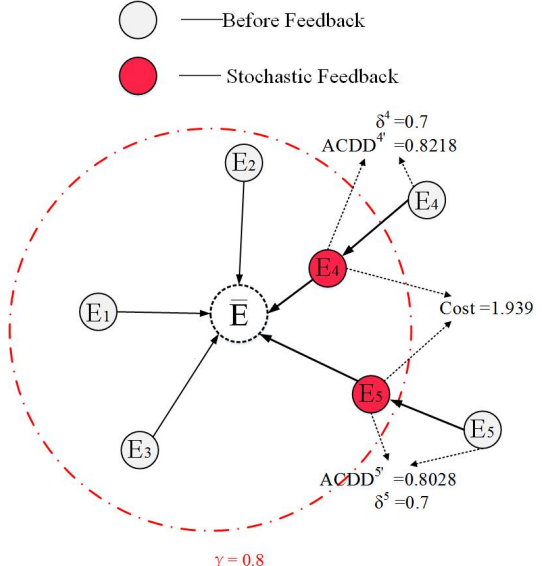

(a) Traditional feedback model

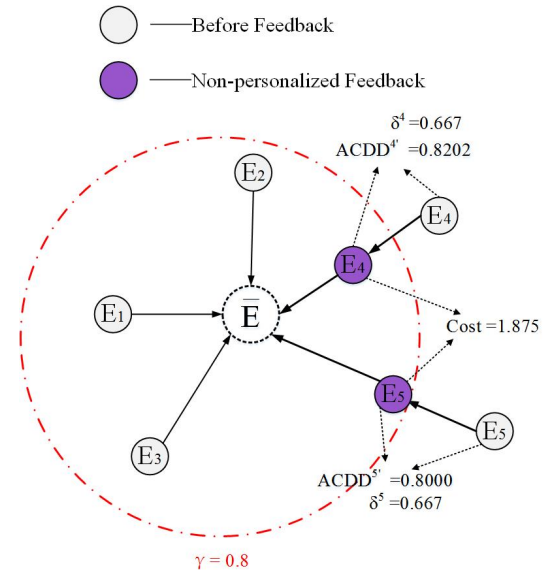

(b) Non-personalized feedback model

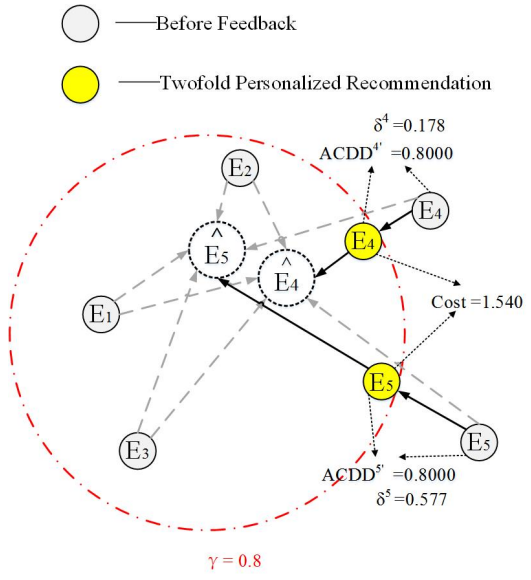

(c) Twofold personalized feedback model

Fig. 3: The comparison of three types of feedback models

\section{REFERENCES}

[1] E. Herrera-Viedma, F. J. Cabrerizo, J. Kacprzyk, and W. Pedrycz, "A review of soft consensus models in a fuzzy environment," Information Fusion, vol. 17, pp. 4-13, 2014.

[2] L. G. Pérez, F. Mata, F. Chiclana, G. Kou, and E. Herrera-Viedma, "Modelling influence in group decision making," Soft Computing, vol. 20, no. 4, pp. 1653-1665, 2016.

[3] Y. T. Liu, Y. C. Dong, H. M. Liang, F. Chiclana, and E. HerreraViedma, "Multiple attribute strategic weight manipulation with minimum cost in a group decision making context with interval attribute weights information," IEEE Transactions on Systems, Man, and Cybernetics: Systems, vol. 49, no. 10, pp. 1981-1992, 2019.

[4] B. Zhu, Z. S. Xu, and J. P. Xu, "Deriving a ranking from hesitant fuzzy preference relations under group decision making," IEEE transactions on Cybernetics, vol. 44, no. 8, pp. 1328-1337, 2014.

[5] R. X. Ding, X. Q. Wang, K. Shang, and F. Herrera, "Social network analysis-based conflict relationship investigation and conflict degreebased consensus reaching process for large scale decision making using sparse representation," Information Fusion, vol. 50, pp. 251-272, 2019.

[6] Z. P. Tian, R. X. Nie, J. Q. Wang, and R. Y. Long, "Adaptive consensusbased model for heterogeneous large-scale group decision making: Detecting and managing non-cooperative behaviors," IEEE Transactions on Fuzzy Systems, 2020. DOI:10.1109/TFUZZ.2020.2995229

[7] R. X. Nie, Z. P. Tian, J. Q. Wang, and H. Y. Luo, "An objective and interactive information based feedback mechanism for the consensus reaching-process considering a non-support degree for minority opinions," Expert Systems, 2020. DOI:10.1111/exsy. 12543

[8] Y. C. Dong and E. Herrera-Viedma, "Consistency-driven automatic methodology to set interval numerical scales of 2-tuple linguistic term sets and its use in the linguistic GDM with preference relation," IEEE transactions on Cybernetics, vol. 45, no. 4, pp. 780-792, 2015.

[9] R. Ureña, F. Chiclana, H. Fujita, and E. Herrera-Viedma, "Confidenceconsistency driven group decision making approach with incomplete reciprocal intuitionistic preference relations," Knowledge-Based Systems, vol. 89, pp. 86-96, 2015.

[10] X. X. Zhang, B. F. Ge, J. Jiang, and Y. J. Tan, "Consensus building in group decision making based on multiplicative consistency with incomplete reciprocal preference relations," Knowledge-Based Systems, vol. 106, pp. 96-104, 2016

[11] M. S. Cao, J. Wu, F. Chiclana, R. Ureña, and E. HerreraViedma, "A personalized feedback mechanism based on maximum harmony degree for consensus in group decision making," IEEE Transactions on Systems, Man, and Cybernetics: Systems, 2020. DOI:10.1109/TSMC.2019.2960052

[12] R. Urena, F. Chiclana, G. Melancon, and E. Herrera-Viedma, "A social network based approach for consensus achievement in multiperson decision making," Information Fusion, vol. 47, pp. 72-87, 2019.

[13] Z. M. Zhang and W. Pedrycz, "A consistency and consensus-based goal programming method for group decision-making with interval-valued intuitionistic multiplicative preference relations," IEEE transactions on cybernetics, vol. 49, no. 10, pp. 3640-3654, 2019.

[14] B. W. Zhang, Y. C. Dong, H. J. Zhang and W. Pedryc, "Consensus Mechanism with Maximum-Return Modifications and Minimum-Cost Feedback: A Perspective of Game Theory," European Journal of Operational Research, vol. 287, no. 2, pp. 546-559, 2020.

[15] J. Wu, R. Y. Xiong, and F. Chiclana, "Uninorm trust propagation and aggregation methods for group decision making in social network with four tuple information," Knowledge-Based Systems, vol. 96, pp. 29-39, 2016.

[16] J. Wu, F. Chiclana, H. Fujita, and E. Herrera-Viedma, "A visual interaction consensus model for social network group decision making with trust propagation," Knowledge-Based Systems, vol. 122, pp. 39-50, 2017.

[17] Y. J. Liu, C. Y. Liang, F. Chiclana, and J. Wu, "A trust induced recommendation mechanism for reaching consensus in group decision making," Knowledge-Based Systems, vol. 119, pp. 221-231, 2017.

[18] Z. B. Wu, B. M. Jin, H. Fujita, and J. P. Xu, "Consensus analysis for AHP multiplicative preference relations based on consistency control: A heuristic approach," Knowledge-Based Systems, vol. 191, p. 105317, 2020.

[19] T. Wu, X. W. Liu, J. D. Qin, and F. Herrera, "Consensus evolution networks: A consensus reaching tool for managing consensus thresholds in group decision making," Information Fusion, vol. 52, pp. 375-388, 2019.

[20] J. Wu, M. S. Cao, F. Chiclana, Y. C. Dong, and E. Herrera-Viedma, "An optimal feedback model to prevent manipulation behaviour in consensus under social network group decision making," IEEE Transactions on Fuzzy Systems, 2020, doi:10.1109/TFUZZ.2020.2985331

[21] H. J. Zhang, S. H. Zhao, G. Kou, C. C. Li, Y. C. Dong, and F. Herrera, "An overview on feedback mechanisms with minimum adjustment or cost in consensus reaching in group decision making: Research paradigms and challenges," Information Fusion, vol. 60, pp. 65-79, 2020.

[22] J. Wu, L. F. Dai, F. Chiclana, H. Fujita, and E. Herrera-Viedma, "A minimum adjustment cost feedback mechanism based consensus model for group decision making under social network with distributed linguistic trust," Information Fusion, vol. 41, pp. 232-242, 2018.

[23] T. T. Gai, M. S. Cao, Q. W. Gao,J. Wu, G. F. Yu, and M. Zhou,"A joint feedback strategy for consensus in large-scale group decision making under social network," Computers \& Industrial Engineering, 2020. DOI:10.1016/j.cie.2020.106626

[24] T. Wu, K. Zhang, X. W. Liu, and C. Y. Cao, "A two-stage social trust network partition model for large-scale group decision-making problems," Knowledge-Based Systems, vol. 163, pp. 632-643, 2019.

[25] Y. D. He, Z. He, and H. Y. Chen, "Intuitionistic fuzzy interaction bonferroni means and its application to multiple attribute decision making," IEEE transactions on cybernetics, vol. 45, no. 1, pp. 116-128, 2015.

[26] L. G. Pérez, F. Mata, and F. Chiclana, "Social network decision making with linguistic trustworthiness-based induced OWA operators," 
International Journal of Intelligent Systems, vol. 29, no. 12, pp. 11171137, 2014.

[27] O. Lesser, L. Naamani-Dery, M. Kalech, and Y. Elovici, "Group decision support for leisure activities using voting and social networks," Group Decision and Negotiation, vol. 26, no. 3, pp. 473-494, 2017.

[28] N. H. Kamis, F. Chiclana, and J. Levesley, "Geo-uninorm consistency control module for preference similarity network hierarchical clustering based consensus model," Knowledge-Based Systems, vol. 162, pp. 103$114,2018$.

[29] N. Capuano, F. Chiclana, E. Herrera-Viedma, H. Fujita, and V. Loia, "Fuzzy group decision making for influence-aware recommendations," Computers in Human Behavior, vol. 101, pp. 371-379, 2019.

[30] Z. W. Gong, H. Wang, W. W. Guo, Z. J. Gong, and H. Wang, "Measuring trust in social networks based on linear uncertainty theory," Information Sciences, vol. 508, pp. 154-172, 2020

[31] P. Victor, C. Cornelis, M. De Cock, and P. P. Da Silva, "Gradual trust and distrust in recommender systems," Fuzzy Sets and Systems, vol. 160, no. 10 , pp. $1367-1382,2009$.

[32] P. Victor, C. Cornelis, M. De Cock, and E. Herrera-Viedma, "Practical aggregation operators for gradual trust and distrust," Fuzzy Sets and Systems, vol. 184, no. 1, pp. 126-147, 2011.

[33] Y. C. Dong, Q. B. Zha, H. J. Zhang, and F. Herrera, "Consensus reaching and strategic manipulation in group decision making with trust relationships," IEEE Transactions on Systems, Man, and Cybernetics: Systems,2020. DOI:10.1109/TSMC.2019.2961752

[34] B. S. Liu, Q. Zhou, R. X. Ding, I. Palomares, and F. Herrera, "Largescale group decision making model based on social network analysis: Trust relationship-based conflict detection and elimination," European Journal of Operational Research, vol. 275, no. 2, pp. 737-754, 2019.

[35] J. Wu, J. L. Chang, Q. W. Cao, and C. Y. Liang, "A trust propagation and collaborative filtering based method for incomplete information in social network group decision making with type-2 linguistic trust," Computers \& Industrial Engineering, vol. 127, pp. 853-864, 2019.

[36] Z. P. Tian, R. X. Nie, and J. Q. Wang, "Social network analysisbased consensus-supporting framework for large-scale group decisionmaking with incomplete interval type-2 fuzzy information," Information Sciences, vol. 502, pp. 446-471, 2019.

[37] J. Wu, X. Li, F. Chiclana, and R. Yager, "An attitudinal trust recommendation mechanism to balance consensus and harmony in group decision making," IEEE Transactions on Fuzzy Systems, vol. 27, no. 11, pp. 2163-2175, 2019

[38] Y. Z. Wu, Y. C. Dong, J. D. Qin, and W. Pedrycz, "Flexible linguistic expressions and consensus reaching with accurate constraints in group decision-making," IEEE transactions on Cybernetics, vol. 50, no. 6, pp. 2488-2501, 2020.

[39] F. Liu, W. Pedrycz, and X. W. Liu, "Flexibility degree of fuzzy numbers and its implication to a group-decision-making model," IEEE transactions on cybernetics, vol. 49, no. 12, pp. 4054-4065, 2018.

[40] H. J. Zhang, Y. C. Dong, J. Xiao, F. Chiclana and E. HerreraViedma,"Personalized individual semantics-based approach for linguistic failure modes and effects analysis with incomplete preference information," IIISE Transactions, Taylor \& Francis Journals, vol. 52, no. 11, pp. 1275-1296, 2020.

[41] G. Alefeld and G. Mayer, "Interval analysis: theory and applications," Journal of Computational and Applied Mathematics, vol. 121, no. 1-2, pp. 421-464, 2000.

[42] R. R. Yager and A. Rybalov, "Uninorm aggregation operators," Fuzzy sets and systems, vol. 80, no. 1, pp. 111-120, 1996.

[43] F. Chiclana, E. Herrera-Viedma, S. Alonso, and F. Herrera, "Cardinal consistency of reciprocal preference relations: a characterization of multiplicative transitivity," IEEE Transactions on fuzzy Systems, vol. 17, no. 1, pp. 14-23, 2009.

[44] A. Sengupta and T. K. Pal, "On comparing interval numbers," European Journal of Operational Research, vol. 127, no. 1, pp. 28-43, 2000.

[45] J. Kacprzyk and M. Fedrizzi, "A soft measure of consensus in the setting of partial (fuzzy) preferences," European Journal of Operational Research, vol. 34, no. 3, pp. 316-325, 1988.

[46] P. Pérez-Asurmendi and F. Chiclana, "Linguistic majorities with difference in support," Applied Soft Computing, vol. 18, pp. 196-208, 2014.

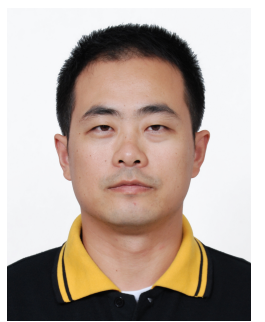

Jian Wu (SM'16) received the B.Sc. and Ph.D. degrees in Management Science and Engineering from Hefei University of Technology, Hefei, China, in 2000 and 2008, respectively. He is a Distinguished Professor with the School of Economics and Management, Shanghai Maritime University, Shanghai, China. He has 80+ papers published in leading journals such IEEE Transactions on Fuzzy Systems, IEEE Transactions on Systems, Man, and Cybernetics:Systems, etc. Fourteen papers have been classed as Highly Cited Papers by the Essential Science Indicators, seven of them are HOT paper. His research interests include group decision making, social network, fuzzy preference modeling, and information fusion. Prof. Wu is an Area Editor of the Journal of Computers \& Industrial Engineering, Associate Editor of the Journal of Intelligent and Fuzzy Systems and a Guest Editor of Applied Soft Computing.

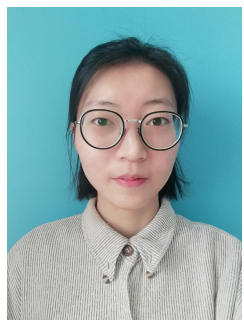

Sha Wang received the BSc in Accounting from Shanghai Maritime University, China, in 2018. She is currently enrolled in a Ph.D. degree at Shanghai Maritime University. Her research interest is mainly in group decision making.

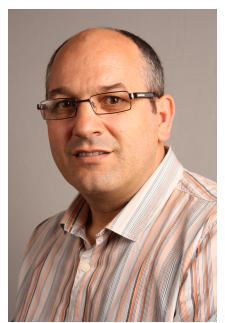

Francisco Chiclana received the BSc and $\mathrm{PhD}$ in Mathematics from the University of Granada, Spain, in 1989 and 2000, respectively.

$\mathrm{He}$ is a Professor of Computational Intelligence and Decision Making with the School of Computer Science and Informatics, Faculty of Computing, Engineering and Media, at De Montfort University, Leicester, U.K. He is currently a Highly Cited Researcher in Computer Sciences (Web of Science Group a Clarivate Analytics company).

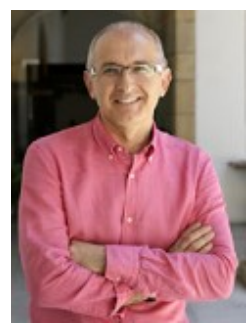

Enrique Herrera-Viedma received the M.Sc. and $\mathrm{Ph} . \mathrm{D}$ degrees in computer science from the University of Granada, Granada, Spain, in 1993 and 1996, respectively. He is a Professor of computer science and the Vice-President for Research and Knowledge Transfer with University of Granada, Granada, Spain. His h-index and sum of citations are: 80 and more than 22000 in Web of Science and 93 and more than 35000 in Google Scholar, respectively. He has been identified as one of the worlds most influential researchers by the Shanghai Center and Thomson Reuters/Clarivate Analytics in both computer science and engineering in the years 2014, 2015, 2016, 2017, 2018 and 2019. His current research interests include group decision making, consensus models, linguistic modeling, aggregation of information, information retrieval,bibliometric, digital libraries, web quality evaluation, recommender systems, and social media. Prof. Herrera-Viedma is currently the Vice-President for Publications for the IEEE SMC Society and an Associate Editor in several journals such as IEEE Transactions on Fuzzy Systems, IEEE Transactions on Systems, Man and Cybernetics: Systems, Information Sciences, Applied Soft Computing, Soft Computing, Fuzzy Optimization and Decision Making, and KnowledgeBased Systems. 